\title{
Erratum to: Temozolomide after radiotherapy in recurrent "low grade" diffuse brainstem glioma in adults
}

\author{
Germán Reyes-Botero • Florence Laigle-Donadey • \\ Karima Mokhtari • Nadine Martin-Duverneuil • \\ Jean-Yves Delattre
}

Published online: 9 October 2014

(c) Springer Science+Business Media New York 2014

\section{Erratum to: J Neurooncol}

\section{DOI 10.1007/s11060-014-1589-9}

In the initial online publication, the authors' family names and first names were interchanged. The names have been corrected in the publication as shown in this erratum.

The online version of the original article can be found under doi:10. 1007/s11060-014-1589-9.

G. Reyes-Botero · F. Laigle-Donadey $(\bowtie) \cdot$ J.-Y. Delattre Service de Neurologie 2, AP-HP, Groupe Hospitalier PitiéSalpêtrière, 47-83 boulevard de l'Hôpital, 75013 Paris, France e-mail: florence.laigle-donadey@psl.aphp.fr

K. Mokhtari

Laboratoire de Neuropathologie Raymond Escourolle AP-HP, Groupe Hospitalier Pitié-Salpêtrière, Paris, France

N. Martin-Duverneuil

Service de Neuroradiologie, AP-HP, Groupe Hospitalier Pitié-

Salpêtrière, Paris, France

\section{J.-Y. Delattre}

Inserm U 1127, CNRS UMR 7225, Sorbonne Universités, UPMC Univ Paris 06 UMR S 1127, Centre de Recherche de L'Institut du Cerveau et de la Moelle épinière (CRICM) UMRS975, Université Pierre et Marie Curie-Paris 6, Paris, France 